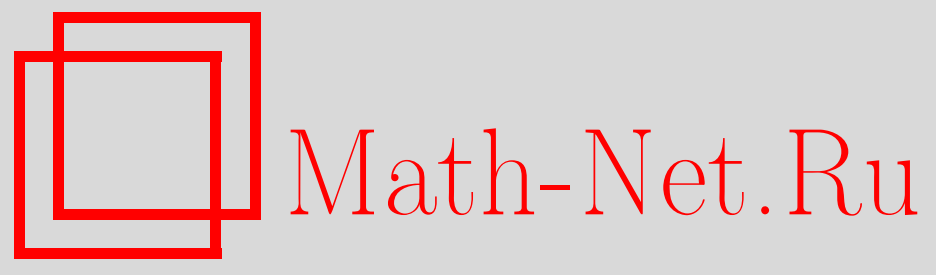

A. Račkauskas, Ch. Suquet, Central limit theorems in Hölder topologies for Banach space valued random fields, Теория вероятн. и ее примен., 2004, том 49, выпуск 1, 109-125

DOI: https://doi.org/10.4213/tvp238

Использование Общероссийского математического портала MathNet.Ru подразумевает, что вы прочитали и согласны с пользовательским соглашением

http://www.mathnet.ru/rus/agreement

Параметры загрузки:

IP : 54.224 .187 .69

26 апреля 2023 г., 12:51:20

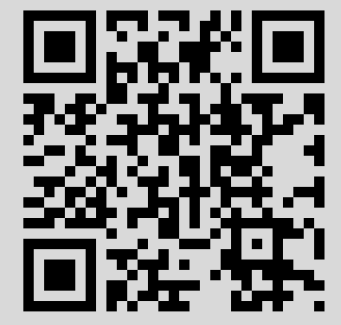




\section{CENTRAL LIMIT THEOREMS IN HÖLDER TOPOLOGIES FOR BANACH SPACE VALUED RANDOM FIELDS ${ }^{1)}$}

Для весьма общих модулей гладкости $\rho$, например, таких, как $\rho(h)=h^{\alpha}:{ }^{\beta}(c / h)$, рассматриваются гёльдеровы пространства $H_{\rho}(B)$ функций из $[0,1]^{d}$ в $B$, где $B$ - сепарабельное банахово пространство. Используя изоморфизм между $H_{\rho}(B)$ и некоторым банаховым пространством последовательностей, мы изучаем в терминах вторых разностей центральную предельную теорему для независимых одинаково распределенных последовательностей случайных элементов в $H_{\rho}(B)$.

Ключевые слова и фразы: броуновское движение со значениями в банаховом пространстве, центральная предельная теорема, неравенство Розенталя, разложение Шаудера, вторая разность, косопирамидальный базис, плотность, пространство типа 2.

\section{Introduction}

Let $\rho(\delta), 0 \leqslant \delta \leqslant 1$, be a modulus of smoothness and $B$ a separable Banach space. Denote by $H_{\rho}(B)=H_{\rho}\left([0,1]^{d} ; B\right)$ the space of all functions $x:[0,1]^{d} \rightarrow B$, such that

$$
\|x(t+h)-x(t)\|_{B}=O(\rho(|h|)),
$$

uniformly in $t \in[0,1)^{d}$. $H_{\rho}^{o}(B)=H_{\rho}^{o}\left([0,1]^{d} ; B\right)$ is the subspace of functions for which $O(\rho(|h|))$ can be replaced by $o(\rho(|h|))$ in (1.1). Equipped with the related Hölder norm (precise definitions are given in Section 2), $H_{\rho}(B)$ becomes a nonseparable Banach space with $H_{\rho}^{o}(B)$ as a closed separable subspace. Letting vary the modulus $\rho$ gives a very natural scale of spaces allowing one to classify by their global regularity the functions more

* Vilnius University, Naugarduko 24, LT-2006, Vilnius, and Institute of Mathematics and Informatics, Akademijos 4, LT-2600, Vilnius, Lithuania; e-mail: alfredas.rackauskas@maf.vu.lt

${ }^{* *}$ Université des Sciences et Technologies de Lille, Statistique et Probabilités, CNRS FRE 2222, Bât. M2, U.F.R. de Mathématiques, F-59655 Villeneuve d'Ascq Cedex, France; e-mail: Charles.Suquet@univ-lille1.fr

1) This research was supported by a cooperation agreement CNRS/LITHUANIA (4714). 
than continuous. This functional framework is interesting in the theory of stochastic process since very often the continuous stochastic process under study have a better regularity than the bare continuity. Moreover for obvious topological reasons, weak convergence in Hölder spaces is stronger than in the classical space $C\left([0,1]^{d} ; B\right)$ of $B$-valued continuous functions on $[0,1]^{d}$. In this paper we establish sufficient conditions for a random element $\xi$ in $H_{\rho}^{o}(B)$ to satisfy the central limit theorem (CLT for short) in this space, i.e., the convergence in distribution of $\zeta_{n}:=n^{-1 / 2}\left(\xi_{1}+\cdots+\xi_{n}\right)$, where the $\xi_{i}$ 's are independent copies of $\xi$.

As far as we know, the first results on the CLT in Hölder spaces are to be found in our recent papers [13] and [14] dealing, respectively, with the case $B=\mathbf{R}, T=[0,1], \rho(h)=|h|^{\alpha}$ and $B=\mathbf{R}, T=[0,1]^{d}$ with general $\rho$. It seems in order to mention here two earlier Hölderian invariance principles for partial sums and for empirical processes which are somewhat related to the problem. In 1962, Lamperti [10] investigated the weak Hölder convergence of Donsker-Prokhorov's polygonal partial sums process (denoted below by FCLT) in the case $B=\mathbf{R}, \rho(h)=|h|^{\alpha}$. This FCLT was extended by Erickson [6] for $B=\mathbf{R}^{k}$ and general $\rho$. Lamperti's result was extended recently by Hamadouche [8] to the case of dependent underlying variables and different smoothings of partial sums. The same author obtained also invariance principles for smooth versions of empirical processes [7]. We discuss in another paper [15] the general problem of FCLT in $H_{\rho}^{o}(B)$ for polygonal process of partial sums of i.i.d. random elements in $B$. Let us point out also a paper of Kerkyacharian and Roynette [9] who emphasized the usefulness of the Faber-Schauder basis of triangular functions in the problem of existence of Hölderian version of a random process as well as for Lamperti's Hölderian FCLT.

The method of triangular functions used by Ciesielski [2], [3], Delporte [5], Kerkyacharian and Roynette [9] relies on the following well-known decomposition of a real-valued continuous function $x$

$$
x(t)=\sum_{j=0}^{\infty} \sum_{v \in V_{j}} \lambda_{j, v}(x) \Lambda_{j, v}(t), \quad t \in T,
$$

where the $\Lambda_{j, v}$ 's are the Faber-Schauder triangular functions and $V_{j}$ is the set of dyadic numbers of level $j$ in $T=[0,1]$. In fact the triangular functions form a basis (in Schauder's sense) of $H_{\rho}^{o}([0,1] ; \mathbf{R})$ when $\rho(h)=|h|^{\alpha}$. Moreover the Hölder regularity of a continuous function is characterized by the rate of decreasing of its coefficients $\lambda_{j, v}(x)$ in this basis. This provides the Ciesielski [2] isomorphisms between these Hölder spaces and some sequence spaces. These isomorphisms give a very convenient discretization procedure in [13] to study the CLT. In [14] we use the same method, replacing the basis of triangular functions by the basis of skew pyramidal functions defined on $T=[0,1]^{d}$, denoted again by $\Lambda_{j, v}$ and indexed by the dyadic points $v$ 
of level $j$ in $[0,1]^{d}$. The scalar coefficients $\lambda_{j, v}(x)$ are some dyadic second differences of $x$. In the present contribution, we keep formally the same decomposition (1.2) of $B$-valued Hölder functions into series of pyramidal functions. But now the pyramidal functions are scalar while their coefficients are vectors living in $B$. The important fact is the preservation in this new setting of the equivalence between the initial Hölder norm $\|x\|_{\rho}$ with the sequential norm

$$
\|x\|_{\rho}^{\text {seq }}:=\sup _{j \geqslant 0} \frac{1}{\rho\left(2^{-j}\right)} \max _{v \in V_{j}}\left\|\lambda_{j, v}(x)\right\|_{B} .
$$

Of course it cannot be question of any Schauder basis for $H_{\rho}^{o}(B)$ in the general case since $B$ itself does not necessarily possess such a basis. Nevertheless the space $H_{\rho}^{o}(B)$ satisfies the more general property of Schauder decomposability (see [19]): it expands in a topological direct sum of a sequence of closed subspaces $\mathbf{W}_{i}$ of piecewise affine functions with values in $B$. This is an useful tool to characterize the tightness of a sequence of random elements in $H_{\rho}^{o}(B)$. Because $B$ may be infinite dimensional, the tightness is not obtained directly here by the classical flat concentration argument.

From (1.2) and (1.3) it should be clear that the control of the coefficients $\lambda_{j, v}(x)$ is the key tool in our study. It is then relevant to state the basic assumptions for the CLT in terms of the second differences

$$
\Delta_{h}^{2} \xi(t):=\xi(t+h)+\xi(t-h)-2 \xi(t)
$$

of the random fields considered. This brings more flexibility than the classical use of first increments.

The paper is organized as follows. Section 2 exposes the analytical preliminaries: expansion in a series of pyramidal functions, equivalence of norms, Schauder decomposability. Section 3 deals with central limit theorem. Of course if the random element $\xi$ satisfies the CLT in $H_{\rho}^{o}(B)$, necessarily $\xi(t)$ and $\Delta_{h}^{2} \xi(t)$ satisfy the CLT in $B$ for each $t$. Because this latter CLT cannot follow in general from any integrability condition on $\xi(t)$, we focus our interest on the following question. If we already know that $\xi(t)$ satisfies the CLT in $B$, which extra conditions imply that $\xi$ satisfies the CLT in $H_{\rho}^{\circ}(B)$ ? This investigation requires the control of normalized sums $n^{-1 / 2} S_{n}$ of independent copies of a random element $X$ in $B$. Their behavior is related to the geometry of $B$ via some moment inequalities involving the special norm $\operatorname{clt}(X):=\sup _{n \geqslant 1} \mathbf{E}\left\|n^{-1 / 2} S_{n}\right\|_{B}$ on the space of random elements satisfying the CLT in $B$. Roughly speaking, our general results says that if $\xi$ satisfies

$$
\mathbf{P}\left\{\left\|\Delta_{h}^{2} \xi(t)\right\|_{B}>r \sigma(|h|)\right\} \leqslant \Psi(r), \quad r>0
$$

and

$$
\operatorname{clt}\left(\Delta_{h}^{2} \xi(t)\right) \leqslant \sigma(|h|)
$$


and if the series

$$
R(u):=\sum_{j=0}^{\infty} 2^{j d} \Psi\left(u \frac{\rho}{\sigma}\left(2^{-j}\right)\right) .
$$

converges for each $u>0$, then $\xi$ verifies the CLT in $H_{\rho}^{o}(B)$. More practical conditions are easily deduced from this general result when $B$ is of type 2 or of cotype 2 or verifies some Rosenthal property. We also prove another CLT in $H_{\rho}^{o}(B)$ when $B$ is of type 2, assuming the square integrability of $\xi$ in some stronger Hölder norm. Finally we discuss the case where $B=C(S)$ the space of continuous functions on a compact metric space. A useful tool in these two last results is the almost sure randomization method.

\section{Analytical background}

The analytical preliminaries exposed in this section are the same as in the paper [17] by the authors. This material is included here for reader's convenience.

Throughout $T=[0,1]^{d}$ and $\mathbf{R}^{d}$ is endowed with the norm

$$
|t|:=\max _{1 \leqslant i \leqslant d}\left|t_{i}\right|, \quad t=\left(t_{1}, \ldots, t_{d}\right) \in \mathbf{R}^{d} .
$$

Denote by $B$ a Banach space with the norm $\|\cdot\|_{B}$ and by $H_{\rho}(B)=H_{\rho}(T ; B)$ the set of $B$-valued continuous functions $x: T \rightarrow B$ such that $\omega_{\rho}(x, 1)<\infty$, where

$$
\omega_{\rho}(x, \delta):=\sup _{t, s \in T, 0<|t-s|<\delta} \frac{\|x(t)-x(s)\|_{B}}{\rho(|s-t|)}
$$

and $\rho$ is a modulus of smoothness satisfying conditions (2.1)-(2.5) below.

The technical conditions required for $\rho$ are the following, where $c_{1}, c_{2}$, and $c_{3}$ are positive constants:

$$
\begin{aligned}
& \rho(0)=0, \quad \rho(\delta)>0, \quad 0<\delta \leqslant 1 ; \\
& \rho \text { is nondecreasing on }[0,1] \text {; } \\
& \rho(2 \delta) \leqslant c_{1} \rho(\delta), \quad 0 \leqslant \delta \leqslant \frac{1}{2} ; \\
& \int_{0}^{\delta} \frac{\rho(u)}{u} d u \leqslant c_{2} \rho(\delta), \quad 0<\delta \leqslant 1 ; \\
& \delta \int_{\delta}^{1} \frac{\rho(u)}{u^{2}} d u \leqslant c_{3} \rho(\delta), \quad 0<\delta \leqslant 1 .
\end{aligned}
$$

To exhibit a concrete class of functions satisfying conditions (2.1)-(2.5), let us define inductively the sequence of functions $\ell_{k}$ on $\left(u_{k}, \infty\right)$ by $\ell_{1}(u)=$ $\ln u, u_{1}=1$ and for $k \geqslant 2$

$$
\ell_{k}(u)=\ln \left(\ell_{k-1}(u)\right)
$$

and $u_{k}$ such that $\ell_{k-1}\left(u_{k}\right)=1$. 
Elementary computations show that for any $\alpha \in(0,1)$ and any finite sequence $\beta_{1}, \ldots, \beta_{m} \in \mathbf{R}$ there exists a set of positive constants $b_{1}, \ldots, b_{m}$ such that the functions

$$
\rho(h)=h^{\alpha} \prod_{k=1}^{m} \ell_{k}^{\beta_{k}}\left(\frac{b_{k}}{h}\right), \quad 0<h \leqslant 1,
$$

satisfy conditions (2.1)-(2.5).

The set $H_{\rho}(B)$ is a Banach space when endowed with the norm $\|x\|_{\rho}:=$ $\|x(0)\|_{B}+\omega_{\rho}(x, 1)$. Obviously an equivalent norm is obtained replacing $\|x(0)\|_{B}$ in the above formula by $\|x\|_{\infty}:=\sup \left\{\|x(t)\|_{B} ; t \in T\right\}$.

Define

$$
H_{\rho}^{o}(B)=H_{\rho}^{o}(T ; B):=\left\{x \in H_{\rho}(B): \lim _{\delta \rightarrow 0} \omega_{\rho}(x, \delta)=0\right\} .
$$

Then $H_{\rho}^{o}(B)$ is a closed subspace of $H_{\rho}(B)$. Now let us remark that for any function $\rho$ satisfying (2.1) and (2.5), there is a positive constant $c_{4}$ such that

$$
\rho(\delta) \geqslant c_{4} \delta, \quad 0 \leqslant \delta \leqslant 1 .
$$

Hence the spaces $H_{\rho}^{o}(B)$ always contain all the Lipschitz $B$-valued functions and in particular the (continuous) piecewise affine functions. When $B$ is itself separable, the separability of the spaces $H_{\rho}^{o}(B)$ follows by interpolation arguments (see Proposition 3).

Since we are interested in the analysis of these spaces in terms of second differences of the functions $x$, our first task is to establish the equivalence of the norm $\|x\|_{\rho}$ with some sequential norm involving the dyadic second differences of $x$. Our main reference for this part is [18].

The so-called skew pyramidal basis was introduced by Bonic, Frampton, and Tromba [1] and independently by Ciesielski and Geba (see the historical notes in $[18$, p. 72$])$. The reader is referred also to our previous contributions [14] and [17] for a more detailed explanation.

If $A$ is a convex subset of $T$, the function $f: T \rightarrow B$ is said to be affine on $A$ if it preserves the barycenter, i.e., for any finite sequence $u_{1}, \ldots, u_{m}$ in $A$ and nonnegative scalars $r_{1}, \ldots, r_{m}$ such that $\sum_{i=1}^{m} r_{i}=1$ we have $f\left(\sum_{i=1}^{m} r_{i} u_{i}\right)=\sum_{i=1}^{m} r_{i} f\left(u_{i}\right)$.

To explain the construction of the skew pyramidal basis, define first the standard triangulation of the unit cube $T=[0,1]^{d}$. Write $\Pi_{d}$ for the set of permutations of the indices $1, \ldots, d$. For any $\pi=\left(i_{1}, \ldots, i_{d}\right) \in \Pi_{d}$, let $\Delta_{\pi}(T)$ be the convex hull of the $d+1$ points $0, e_{i_{1}},\left(e_{i_{1}}+e_{i_{2}}\right), \ldots, \sum_{k=1}^{d} e_{i_{k}}$, where $e_{i}$ 's are the vectors of the canonical basis of $\mathbf{R}^{d}$. So, each simplex $\Delta_{\pi}(T)$ corresponds to one path from 0 to $(1, \ldots, 1)$ via vertices of $T$ and such that along each segment of the path, only one coordinate increases while the others remain constants. Thus $T$ is divided into $d$ ! simplexes with disjoint interiors. The standard triangulation of $T$ is the family $T_{0}$ of simplexes $\left\{\Delta_{\pi}(T)\right.$, $\left.\pi \in \Pi_{d}\right\}$. 
Next, we divide $T$ into $2^{j d}$ dyadic cubes with edge $2^{-j}$. By dyadic translations and change of scale, each of them is equipped with a triangulation similar to $T_{0}$. And $T_{j}$ is the set of the $2^{j d} d$ ! simplexes so constructed.

Introduce for $j \geqslant 1$, the set

$$
W_{j}=\left\{k 2^{-j} ; 0 \leqslant k \leqslant 2^{j}\right\}^{d} .
$$

In what follows we put $V_{0}:=W_{0}$ and $V_{j}:=W_{j} \backslash W_{j-1}$. So $V_{j}$ is the set of dyadic points $v=\left(k_{1} 2^{-j}, \ldots, k_{d} 2^{-j}\right)$ in $W_{j}$ with at least one $k_{i}$ odd.

The pyramidal functions $\Lambda_{j, v}$ are defined by

$$
\Lambda_{j, v}(t)=\Lambda\left(2^{j}(t-v)\right)_{\bullet} \quad|t-v| \leqslant 2^{-j},
$$

where $\Lambda(t):=\max \left(0,1-\max _{t_{i}<0}\left|t_{i}\right|-\max _{t_{i}>0} t_{i}\right), t=\left(t_{1}, \ldots, t_{d}\right) \in \mathbf{R}^{d}$.

The $B$-valued coefficients $\lambda_{j, v}(x)$ are given by

$$
\begin{array}{ll}
\lambda_{0, v}(x)=x(v), & v \in V_{0} ; \\
\lambda_{j, v}(x)=x(v)-\frac{1}{2}\left(x\left(v^{-}\right)+x\left(v^{+}\right)\right), & v \in V_{j}, \quad j \geqslant 1,
\end{array}
$$

where $v^{-}$and $v^{+}$are defined as follows. Each $v \in V_{j}$ admits a unique representation $v=\left(v_{1}, \ldots, v_{d}\right)$ with $v_{i}=k_{i} 2^{-j}(1 \leqslant i \leqslant d)$. The points $v^{-}=\left(v_{1}^{-}, \ldots, v_{d}^{-}\right)$and $v^{+}=\left(v_{1}^{+}, \ldots, v_{d}^{+}\right)$are defined by

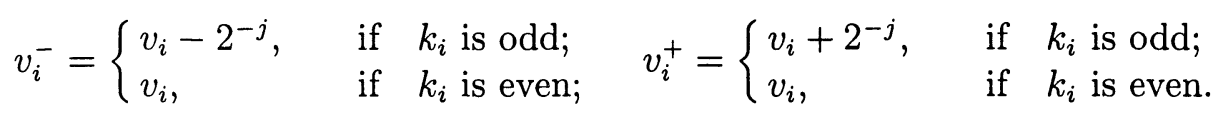

Since $v$ is in $V_{j}$, at least one of the $k_{i}$ 's is odd, so $v^{-}, v$, and $v^{+}$are really three distinct points of $T$. Moreover we can write $v^{-}=v-2^{-j} e(v), v^{+}=$ $v+2^{-j} e(v)$ with $e(v):=\sum_{k_{i} \text { odd }} e_{i}$, so $\lambda_{j, v}(x)$ is a second difference directed by the vector $e(v)$. Observe moreover that if $v$ is in $V_{j}$, then $v^{-}$and $v^{+}$are in $W_{j-1}$.

As usual the space $C(T ; B)$ of continuous functions $x: T \rightarrow B$ is endowed with the uniform norm $\|x\|_{\infty}=\sup _{t \in T}\|x(t)\|_{B}$. Define the operators $E_{j}(j \geqslant 0)$ on the space $C(T ; B)$ of continuous $B$-valued functions by

$$
E_{j} x:=\sum_{i=0}^{j} \sum_{v \in V_{i}} \lambda_{i, v}(x) \Lambda_{i, v}, \quad x \in C(T ; B) .
$$

Then

$$
\left\|x-E_{J} x\right\|_{\rho}^{\text {seq }}:=\sup _{j>J} \frac{1}{\rho\left(2^{-j}\right)} \max _{v \in V_{j}}\left\|\lambda_{j, v}(x)\right\|_{B},
$$

is nonincreasing in $J$.

The proof of the following lemma is elementary (details can be found in [16]).

Lemma 1. The B-valued function $E_{j} x$ is affine on each simplex of $T_{j}$ and such that $E_{j} x(w)=x(w)$ for each $w \in W_{j}$. 
Since the pyramidal functions are not $B$-valued, they cannot form a Schauder basis of $C(T ; B)$ as in the real-valued case. Nevertheless we keep the same type of decomposition.

Proposition 1. Each $x$ in $C(T ; B)$ admits the series expansion

$$
x(t)=\sum_{j=0}^{\infty} \sum_{v \in V_{j}} \lambda_{j, v}(x) \Lambda_{j, v}(t), \quad t \in T,
$$

where the convergence holds in the strong topology of $B$ and is uniform with respect to $t$ on $T$.

For any function $x:[0,1]^{d} \rightarrow B$ the (possibly infinite) sequential seminorm is defined by

$$
\|x\|_{\rho}^{\text {seq }}:=\sup _{j \geqslant 0} \frac{1}{\rho\left(2^{-j}\right)} \max _{v \in V_{j}}\left\|\lambda_{j, v}(x)\right\|_{B} .
$$

Observe moreover that when $x$ is continuous, $\|x\|_{\rho}^{\text {seq }}=0$ if and only if $x=0$. In this case, $\|x\|_{\rho}^{\text {seq }}$ is a (possibly infinite) true norm.

Proposition 2. Under conditions (2.1)-(2.5), the norm $\|x\|_{\rho}$ is equivalent on $C(T ; B)$ to the sequential norm, i.e., there are positive constants $a, b$ such that for every $x \in C(T ; B)$,

$$
a\|x\|_{\rho}^{\text {seq }} \leqslant\|x\|_{\rho} \leqslant b\|x\|_{\rho}^{\text {seq }}
$$

with finite values of the norms if and only if $x \in H_{\rho}(B)$.

The proof is exactly the same as for [14, Proposition 1] with substitution of $|x(t)|$ instead of $\|x(t)\|_{B}$. Note that in this proof the continuity of $x$ was used through its expansion in a series of pyramidal functions which is now replaced by Proposition 1. Conditions (2.4) and (2.5) are not required for the inequality $a\|x\|_{\rho}^{\text {seq }} \leqslant\|x\|_{\rho}$ which holds true with $a=\min (1, \rho(1))$ for the most general moduli of smoothness $\rho$.

As we observed above, the skew pyramidal basis is trivially not a Schauder basis of $H_{\rho}^{o}(B)$. In fact it is even possible that $H_{\rho}^{o}(B)$ could have no Schauder basis at all. It is known since 1972 (see [4]) that there exists separable Banach spaces without any Schauder basis. If $B$ is such a space, then $H_{\rho}^{o}(B)$ cannot have any Schauder basis since it always contains the constant $B$-valued functions and convergence of series in $H_{\rho}^{o}(B)$ implies the pointwise convergence in the strong topology of $B$. Fortunately the more general property of Schauder decomposability is preserved. This property is useful to characterize the tightness of a sequence of random elements in $H_{\rho}^{o}(B)$. Recall that a Banach space $\mathscr{X}$ admits the Schauder decomposition $\left(\mathscr{X}_{i}, i \in \mathbf{N}\right)$ if $\mathscr{X}_{i}^{\prime}$ 's are closed nontrivial subspaces of $\mathscr{X}$ and if each $x \in \mathscr{X}$ admits a unique series expansion $x=\sum_{i=0}^{\infty} x_{i}$ with $x_{i} \in \mathscr{X}_{i}$, convergence in the strong topology of $\mathscr{X}$ and continuity of the projectors $x \mapsto x_{i}$. In other words, $\mathscr{X}$ is the direct topological sum of the $\mathscr{X}_{i}$ 's. 
Proposition 3. The space $H_{\rho}^{o}(B)$ has the Schauder decomposition

$$
H_{\rho}^{o}(B)=\bigoplus_{i=0}^{\infty} \mathbf{W}_{i} .
$$

Here $\mathbf{W}_{0}$ is the space of $B$-valued functions continuous on $T$ and affine on each simplex $\Delta$ of $T_{0}$. For $i \geqslant 1, \mathbf{W}_{i}$ is the space of $B$-valued functions continuous on $T$, affine on each simplex $\Delta$ of $T_{i}$ and vanishing at the vertices of $W_{i-1}$.

$\mathrm{P}$ r o o f. Since convergence in $H_{\rho}^{o}(B)$ implies the uniform convergence on $T$, each $\mathbf{W}_{i}$ is obviously a closed (infinite dimensional) subspace of $H_{\rho}^{o}(B)$. By Proposition 1, each $x$ in $H_{\rho}^{o}(B)$ admits a series expansion $x=\sum_{i=0}^{\infty} x_{i}$, where $x_{i}:=\left(E_{i}-E_{i-1}\right) x$ belongs to $\mathbf{W}_{i}$ and the convergence holds at least in the $C(T ; B)$ sense. In fact, using the obvious estimate $\left\|x-E_{j} x\right\|_{\rho}^{\text {seq }} \leqslant$ $\omega_{\rho}\left(x, 2^{-j}\right)$, we see that this convergence holds also in the strong topology of $H_{\rho}^{o}(B)$.

Suppose now we have another decomposition $x=\sum_{i=0}^{\infty} y_{i}, y_{i} \in \mathbf{W}_{i}$, with convergence in $H_{\rho}^{o}(B)$. Then we have a fortiori $\sum_{i=0}^{\infty} x_{i}(t)=\sum_{i=0}^{\infty} y_{i}(t)$ with convergence in $B$ for each $t$ in $T$. From the definition of $\mathbf{W}_{i}$ 's, we see that if $w \in W_{j}$ and $i>j$, then $x_{i}(w)=y_{i}(w)=0$. Hence $\sum_{i=0}^{j} x_{i}(w)=\sum_{i=0}^{j} y_{i}(w)$, $w \in W_{j}, j \geqslant 0$. So the functions $\sum_{i \leqslant j} x_{i}$ and $\sum_{i \leqslant j} y_{i}$ are affine on each simplex $\Delta$ of $T_{j}$ and coincide at the vertices of $\Delta$. Then they coincide everywhere on $\Delta$. The unicity of the expansion of $x$ follows.

Finally the inequality $\left\|E_{i} x-E_{i-1} x\right\|_{\rho}^{\text {seq }} \leqslant\|x\|_{\rho}^{\text {seq }}$ gives the continuity of the projectors $x \mapsto x_{i}$.

\section{Central limit theorem in $H_{\rho}^{o}(B)$}

We now turn to the central limit theorem for random elements in the separable space $H_{\rho}^{o}(B)$. We shall study the case of i.i.d. sequences. As a basic tool in this problem, we need some tightness results we are beginning with.

3.1. Tightness in $H_{\rho}^{o}(B)$. We first investigate conditions for the tightness of a sequence of random elements in $H_{\rho}^{o}(B)$. Our basic tool is the following.

Theorem 1 (see [20]). Let $\mathscr{X}$ be a separable Banach space having the Schauder decomposition $\mathscr{X}=\oplus_{i=0}^{\infty} \mathscr{X}_{i}$. Write $\mathscr{Z}_{j}=\oplus_{i=0}^{j} \mathscr{X}_{i}$ and denote by $E_{j}$ the projection of $\mathscr{X}$ onto $\mathscr{Z}_{j}$. A sequence of probability measures $\left(\mu_{n}\right)_{n \geqslant 1}$ on the Borel $\sigma$-field of $\mathscr{X}$ is tight if and only if it satisfies both following conditions:

(i) For each $j \geqslant 0$, the sequence of image measures $\left(\mu_{n} \circ E_{j}^{-1}\right)_{n \geqslant 1}$ is tight on $\mathscr{Z}_{j}$;

(ii) for each positive $\varepsilon$,

$$
\lim _{j \rightarrow \infty} \sup _{n \geqslant 1} \mu_{n}\left(\left\{x \in \mathscr{X} ;\left\|x-E_{j} x\right\|>\varepsilon\right\}\right)=0 .
$$


As an application we have the following tightness criterion.

Theorem 2. Suppose the Banach space $B$ is separable. Then the sequence $\left(\xi_{n}\right)_{n \geqslant 1}$ of random elements in $H_{\rho}^{o}(B)$ is tight if and only if it satisfies the two following conditions:

(i) For each dyadic $t \in[0,1]^{d}$, the sequence of $B$-valued random variables $\left(\xi_{n}(t)\right)_{n \geqslant 1}$ is tight on $B$ :

(ii) for each positive $\varepsilon$,

$$
\lim _{j \rightarrow \infty} \sup _{n \geqslant 1} \mathbf{P}\left\{\left\|\xi_{n}-E_{j} \xi_{n}\right\|_{\rho}^{\text {seq }}>\varepsilon\right\}=0 .
$$

P r o of. The necessity of (i) follows from the continuity on $H_{\rho}^{o}(B)$ of the evaluation functionals $x \mapsto x(t)$. Let us check that condition (i) Theorem 2 implies Theorem 1 (i) when $\mathscr{X}=H_{\rho}^{o}(B)$. Write $\mathbf{V}_{j}:=\oplus_{i=0}^{j} \mathbf{W}_{i}$ for the space of $B$-valued functions continuous on $T$ and affine on each simplex $\Delta$ of $T_{j}$. The projector $E_{j}$ is then the operator of affine interpolation at the vertices of $W_{j}$. It is easily seen that $\mathbf{V}_{j}$ is isomorphic to the Cartesian product of $\operatorname{card}\left(\mathrm{W}_{\mathrm{j}}\right)$ copies of $B$. By Prokhorov's theorem and the sequential characterization of relative compactness, Theorem 1 (i) follows now clearly from Theorem 2 (i).

Having in mind Proposition 3 and Proposition 2, we see immediately that (3.1) is a simple translation of (ii) in Theorem 1.

$\mathrm{R}$ e $\mathrm{m}$ a r $\mathrm{k} 1$. We may replace (3.1) by the equivalent condition

$$
\lim _{j \rightarrow \infty} \limsup _{n \rightarrow \infty} \mathbf{P}\left\{\left\|\xi_{n}-E_{j} \xi_{n}\right\|_{\rho}^{\text {seq }}>\varepsilon\right\}=0 .
$$

Indeed, let $\left(u_{n, j}\right)_{n \geqslant 1, j \geqslant 1}$ be a multisequence of real numbers. If for each fixed $n, \quad\left(u_{n, j}\right)_{j \geqslant 1}$ is nonincreasing with limit 0 and if $\lim _{j \rightarrow \infty} \lim \sup _{n \rightarrow \infty} u_{n, j}=0$, then $\lim _{j \rightarrow \infty} \sup _{n \geqslant 1} u_{n, j}=0$. Putting $u_{n, j}:=$ $\mathbf{P}\left\{\left\|\xi_{n}-E_{j} \xi_{n}\right\|_{\rho}^{\text {seq }}>\varepsilon\right\}$ gives the equivalence of (3.1) and (3.2).

$\mathrm{R} \mathrm{e} \mathrm{m} \mathrm{a} \mathrm{r} \mathrm{k} \mathrm{2.} \mathrm{If} B$ is itself Schauder decomposable, Theorem 1 can be used again to check condition (i) of Theorem 2 .

Corollary 1. Let $\left(\xi_{n}\right)_{n \geqslant 1}$ be a sequence of random elements in $H_{\rho}^{o}(B)$ satisfying (i) of Theorem 2. Suppose moreover that

$$
\mathbf{P}\left\{\left\|\Delta_{h}^{2} \xi_{n}(t)\right\|_{B}>r \sigma(|h|)\right\} \leqslant \Psi(r),
$$

with the same $\sigma$ and $\Psi$ independent of $n$. If $\sum_{j=0}^{\infty} 2^{j d} \Psi\left(u \rho \sigma^{-1}\left(2^{-j}\right)\right)<\infty$ for each $u>0$, then $\left(\xi_{n}\right)_{n \geqslant 1}$ is tight in $H_{\rho}^{o}(B)$.

3.2. CLT in the i.i.d. case. For a random element $\xi$ in the Banach space $\mathscr{X}$, we denote by $\xi_{1}, \ldots, \xi_{n}$ independent copies of $\xi$ and

$$
\zeta_{n}=\zeta_{n}(\xi):=n^{-1 / 2} \sum_{k=1}^{n} \xi_{k}
$$


We say that $\xi$ satisfies the central limit theorem in $\mathscr{X}$ if the sequence $\left(\zeta_{n}\right)$ converges in distribution in $\mathscr{X}$. This property will be denoted by $\xi$ satisfies $\operatorname{CLT}(\mathscr{X})$ or $\xi \in \operatorname{CLT}(\mathscr{X})$. We investigate in this section the case $\mathscr{X}=H_{\rho}^{\circ}(B)$ with $B$ separable.

For $X$ random element in the Banach space $B$, let us define

$$
\operatorname{clt}(X):=\sup _{n \geqslant 1} \mathbf{E}\left\|n^{-1 / 2} S_{n}\right\|_{B}
$$

where $S_{n}$ is the sum of $n$ independent copies of $X$. It is well known that if $X \in \operatorname{CLT}(B), \operatorname{clt}(X)$ is finite and that $\operatorname{clt}(\cdot)$ is a norm on the space of all random elements satisfying $\operatorname{CLT}(B)$ (cf., for instance, [12]).

Since $H_{\rho}^{\circ}(B)$ is a subspace of the Cartesian product $B^{T}$ of Polish spaces (i.e., the space of all functions $x: T \rightarrow B$ ), any probability measure on the Borel sigma-field of $H_{\rho}^{o}(B)$ is characterized by its finite dimensional distributions. Hence $\left(\zeta_{n}\right)_{n \geqslant 1}$ converges weakly in $H_{\rho}^{o}(B)$ if and only if it is tight and the random vectors $Z_{n, t_{1} \cdots t_{m}}=\left(\zeta_{n}\left(t_{1}\right), \ldots, \zeta_{n}\left(t_{m}\right)\right)$ converge weakly in $B^{m}$ for every $m \geqslant 1$ and $t_{1}, \ldots, t_{m} \in T$. The expression convergence of finite dimensional distributions is somewhat improper here because the random vector $Z_{n, t_{1} \cdots t_{m}}$ lives in the infinite dimensional Banach space $B^{m}$. So the convergence of finite dimensional distributions in the proof of the CLT in $H_{\rho}^{o}(B)$ already involves some CLT in an infinite dimensional Banach space. A preliminary simplification is the reduction of this problem to the case $m=1$.

Lemma 2. Assume that $\xi(t) \in \operatorname{CLT}(B)$ for each $t \in T$. Then $X_{t_{1} \cdots t_{m}}:=\left(\xi\left(t_{1}\right), \ldots, \xi\left(t_{m}\right)\right)$ satisfies $\operatorname{CLT}\left(B^{m}\right)$ for every $t_{1}, \ldots, t_{m} \in T$.

$\mathrm{P}$ r o o f. Because $B^{m}$ is equipped with the product topology, the tightness of the sequence of random elements $Z_{n, t_{1} \cdots t_{m}}$ in $B^{m}$ follows from the tightness in $B$ of the $m$ sequences $\left(\zeta_{n}\left(t_{i}\right)\right)_{n \geqslant 1}$. Next, denoting by $f$ any linear continuous functional on $B^{m}$, we have to prove the weak convergence of $f\left(Z_{n, t_{1} \cdots t_{m}}\right)$ to $f(G)$, where $G$ is a Gaussian random element in $B^{m}$. By linearity

$$
f\left(Z_{n, t_{1} \cdots t_{m}}\right)=n^{-1 / 2} \sum_{k=1}^{n} f\left(X_{k, t_{1} \cdots t_{m}}\right),
$$

so the problem is reduced to the (i.i.d.) CLT on $\mathbf{R}$ and the only thing to check is the finiteness of $\mathbf{E} f^{2}\left(X_{t_{1} \cdots t_{m}}\right)$. Write $f_{i}\left(x_{i}\right)=f\left(0, \ldots, 0, x_{i}, 0, \ldots, 0\right)$ and observe that $f_{i}$ so defined belongs to $B^{\prime}$. Since each $\xi\left(t_{i}\right)$ is pre-Gaussian in $B, \mathbf{E} f_{i}^{2}\left(\xi\left(t_{i}\right)\right)<\infty$. The proof is completed with the obvious decomposition $f^{2}\left(X_{t_{1} \cdots t_{m}}\right)=\sum_{i, j \leqslant m} f_{i}\left(\xi\left(t_{i}\right)\right) f_{j}\left(\xi\left(t_{j}\right)\right)$.

We begin with a general CLT without any assumption on the properties of the separable Banach space $B$.

Theorem 3. Let $\phi$ be one of the following Young functions: $\phi(r)=r^{p}$ $(p \geqslant 2) ; \phi(r)=\phi_{\gamma}(r)=\exp \left(r^{\gamma}\right)-1,0<\gamma \leqslant 2$. Let $\xi$ be an $H_{\rho}^{o}(B)$-valued random field. Assume that 
(i) for every $t \in T, \xi(t) \in \mathrm{CLT}(B)$;

(ii) there exists a function $\sigma:[0,1] \rightarrow \mathbf{R}, \sigma(0)=0$, such that for all $t \in T$ and $h \in C_{t}$,

$$
\left\|\Delta_{h}^{2} \xi(t)\right\|_{\phi} \leqslant \sigma(|h|)
$$

(iii)

$$
\operatorname{clt}\left(\Delta_{h}^{2} \xi(t)\right) \leqslant \sigma(|h|)
$$

(iv) for $\phi(r)=r^{p}$,

$$
\sum_{j=0}^{\infty} \frac{2^{j d} \sigma^{p}\left(2^{-j}\right)}{\rho^{p}\left(2^{-j}\right)}<\infty
$$

while for $\phi(r)=\phi_{\gamma}(r)$,

$$
\lim _{j \rightarrow \infty} \frac{\rho\left(2^{-j}\right)}{j^{1 / \gamma} \sigma\left(2^{-j}\right)}=\infty
$$

Then $\xi$ satisfies $\operatorname{CLT}\left(H_{\rho}^{\circ}(B)\right)$.

P r o o f. From (i) and Lemma 2 it is easy to see that $\Delta_{h}^{2} \xi(t)$ satisfies CLT $(B)$, so condition (iii) takes sense.

The convergence of finite dimensional distributions of $\zeta_{n}$ follows from (i) and Lemma 2. As a by-product we have also the tightness in $B$ of the sequence $\left(\zeta_{n}(t)\right)_{n \geqslant 1}$ for each dyadic $t$. So to establish the tightness of $\left(\zeta_{n}\right)_{n \geqslant 1}$ in $H_{\rho}^{o}(B)$, it suffices to verify the series condition of Corollary 1. To this end, we use the following set of moment inequalities, where we write $S_{n}$ for the sum of i.i.d. random elements $X_{1}, \ldots, X_{n}$ in $B$. These inequalities are valid for any separable Banach space $B$.

By [12, Theorem 6.20], there is a constant $K$ depending only on $p$ such that

$$
\left(\mathbf{E}\left\|S_{n}\right\|_{B}^{p}\right)^{1 / p} \leqslant K\left(\mathbf{E}\left\|S_{n}\right\|_{B}+\left[\mathbf{E}\left(\max _{1 \leqslant k \leqslant n}\left\|X_{k}\right\|_{B}\right)^{p}\right]^{1 / p}\right) .
$$

Similarly [12, Theorem 6.21] gives a constant $K$ depending only on $\gamma$ such that if $0<\gamma \leqslant 1$, then

$$
\left\|S_{n}\right\|_{\phi_{\gamma}} \leqslant K\left(\mathbf{E}\left\|S_{n}\right\|_{B}+\left\|\max _{1 \leqslant k \leqslant n}\right\| X_{k}\left\|_{B}\right\|_{\phi_{\gamma}}\right),
$$

and if $1<\gamma \leqslant 2$, with $\gamma^{\prime}=\gamma /(\gamma-1)$, then

$$
\left\|S_{n}\right\|_{\phi_{\gamma}} \leqslant K\left(\mathbf{E}\left\|S_{n}\right\|_{B}+\left(\sum_{k=1}^{n}\left\|X_{k}\right\|_{\phi_{\gamma}}^{\gamma^{\prime}}\right)^{1 / \gamma^{\prime}}\right) .
$$

Since $X_{k}$ 's are identically distributed, the second terms in the right-hand sides of (3.3) and (3.5) are bounded, respectively, by $n^{1 / p}\left(\mathbf{E}\left\|X_{1}\right\|_{B}^{p}\right)^{1 / p}$ and $n^{1 / \gamma^{\prime}}\left\|X_{1}\right\|_{\phi_{\gamma}}$ with $p \geqslant 2$ and $\gamma^{\prime} \geqslant 2$. As for the second term in the righthand side of (3.4), using elementary techniques mimicking the classical estimate of maximum for i.i.d. sub-Gaussian random variables, we obtain 
$\left\|\max _{1 \leqslant k \leqslant n}\right\| X_{k}\left\|_{B}\right\|_{\phi_{\gamma}} \leqslant c(\ln n)^{1 / \gamma}\left\|X_{1}\right\|_{\phi_{\gamma}}$ for some constant $c$. When applied to $X_{k}=n^{-1 / 2} \Delta_{h}^{2} \xi_{k}(t)$, the moment inequalities (3.3), (3.4), and (3.5) provide then the unified form estimate

$$
\left\|\Delta_{h}^{2} \zeta_{n}(t)\right\|_{\phi} \leqslant K\left(\operatorname{clt}\left(\Delta_{h}^{2} \xi(t)\right)+u_{n}\left\|\Delta_{h}^{2} \xi(t)\right\|_{\phi}\right),
$$

where

$$
u_{n}=\left\{\begin{array}{lll}
n^{1 / p-1 / 2} & \text { when } \phi(r)=r^{p}, p \geqslant 2 ; \\
c n^{-1 / 2}(\ln n)^{1 / \gamma} & \text { when } \phi=\phi_{\gamma}, 0<\gamma \leqslant 1 ; \\
n^{1 / \gamma^{\prime}-1 / 2} & \text { when } \phi=\phi_{\gamma}, 1<\gamma \leqslant 2 .
\end{array}\right.
$$

Hence in each case $u_{n}$ is bounded, so applying (ii) and (iii) we have $\left\|\Delta_{h}^{2} \zeta_{n}(t)\right\|_{\phi} \leqslant K^{\prime} \sigma(|h|)$ for some constant $K^{\prime}$. Finally one easily checks that the series condition of Corollary 1 is satisfied.

Theorem 3 is of rather theoretical nature. Its main interest is perhaps to separate clearly a condition concerning the geometry of the underlying space $B$ and a condition on the tail behavior of the second differences of $\xi$. In general conditions (i) and (iii) seem not very tractable. The situation becomes much better when $B$ has type 2 or cotype 2 or satisfies the Rosenthal property $\operatorname{Ros}(q)$ for some $q>2$. Recall that a random element $X$ in $B$ such that for every $f$ in $B^{\prime}, \mathbf{E} f(X)=0$ and $\mathbf{E} f^{2}(X)<\infty$ is said to be preGaussian if there exists a Gaussian random element $G(X)$ in $B$ with the same covariance structure as $X$. The separable Banach space $B$ satisfies $\operatorname{Ros}(q)$, $1 \leqslant q<\infty$, if there is a constant $C$ such that for any finite sequence $\left(X_{i}\right)$ of independent pre-Gaussian random elements in $B$ with associated Gaussian variables $\left(G\left(X_{i}\right)\right)$ (which may be assumed to be independent)

$$
\mathbf{E}\left\|\sum_{i} X_{i}\right\|^{q} \leqslant C\left(\sum_{i} \mathbf{E}\left\|X_{i}\right\|^{q}+\mathbf{E}\left\|\sum_{i} G\left(X_{i}\right)\right\|^{q}\right) .
$$

For all these properties we refer to [12].

Corollary 2. In the three following special cases, Theorem 3 remains valid with assumptions (i) and (iii) modified in the following way:

1) When $B$ is of type 2 ,

(a) replace (i) by $\mathbf{E} \xi(t)=0$ and $\mathbf{E}\|\xi(t)\|_{B}^{2}<\infty$,

(b) suppress (iii).

2) When $B$ is of cotype 2 ,

(a) replace (i) by $\xi(t)$ pre-Gaussian;

(b) replace (iii) by $\left(\mathbf{E}\left\|G\left(\Delta_{h}^{2} \xi(t)\right)\right\|^{2}\right)^{1 / 2} \leqslant \sigma(|h|)$.

3) When $B$ satisfies the property $\operatorname{Ros}(q)$ for some $q>2$,

(a) replace (i) by $\xi(t)$ pre-Gaussian and $\lim _{\lambda \rightarrow \infty} \lambda^{2} \mathbf{P}\left\{\|\xi(t)\|_{B}>\lambda\right\}=0$;

(b) replace (iii) by $\left(\mathbf{E}\left\|G\left(\Delta_{h}^{2} \xi(t)\right)\right\|\right)^{1 / 2} \leqslant \sigma(|h|)$.

$\mathrm{P}$ r o o f. When $B$ is of type 2 , under (a), we have $\xi(t) \in \operatorname{CLT}(B)$ by [12, Theorem 10.5]. Moreover $\mathbf{E} \Delta_{h}^{2} \xi(t)=0$ and $\mathbf{E}\left\|\Delta_{h}^{2} \xi(t)\right\|_{B}^{2}<\infty$, so by [12, Proposition 9.11], $\operatorname{clt}\left(\Delta_{h}^{2} \xi(t)\right) \leqslant C\left(\mathbf{E}\left\|\Delta_{h}^{2} \xi(t)\right\|_{B}^{2}\right)^{1 / 2} \leqslant C^{\prime}\left\|\Delta_{h}^{2} \xi(t)\right\|_{\phi}$, for 
each Young function $\phi$ considered in Theorem 3. Hence in this special case, (iii) follows from (ii).

When $B$ is of cotype 2, every pre-Gaussian random element satisfies $\operatorname{CLT}(B)$ (see [12, Theorem 10.7] ), so (i) follows from (a). Moreover by Lemma $2, \Delta_{h}^{2} \xi(t) \in \operatorname{CLT}(B)$, so it is also pre-Gaussian. By the beginning of the proof of [12, Theorem 10.7], we have

$$
\operatorname{clt}\left(\Delta_{h}^{2} \xi(t)\right) \leqslant\left(\mathbf{E}\left\|G\left(\Delta_{h}^{2} \xi(t)\right)\right\|^{2}\right)^{1 / 2},
$$

so (iii) follows from (b).

When $B$ satisfies $\operatorname{Ros}(q)$ for some $q>2$, (a) implies $\xi(t) \in \operatorname{CLT}(B)$. Then, as in the previous case, $\Delta_{h}^{2} \xi(t)$ is pre-Gaussian. By a standard symmetrization argument (see, e.g., [12, p. 279]) we may assume $\xi$ symmetric. Then inequality (10.7) in [12, p. 285] writes here $\operatorname{clt}\left(\Delta_{h}^{2} \xi(t)\right) \leqslant$ $C\left(\left\|\Delta_{h}^{2} \xi(t)\right\|_{2, \infty}+\mathbf{E}\left\|G\left(\Delta_{h}^{2} \xi(t)\right)\right\|_{B}\right)$. Since any $\phi$-Orlicz norm considered in Theorem 3 is stronger than $\|\cdot\|_{2, \infty}$, (iii) follows from (b) and (ii).

Our next result deals with the special case of type 2 spaces $B$. Roughly speaking, the idea is to assume the square integrability of $\xi$ in some stronger Hölder norm than $\|\cdot\|_{\rho}$. A useful tool to prove the corresponding Hölderian CLT is the following special case of the almost sure randomized version of the CLT of Ledoux Talagrand and Zinn. We state it here in a form adapted to our setting for the convenience of reference.

Theorem 4 ([12, Theorem 10.14]). Let $X$ be a mean zero Borel random variable with values in a separable Banach space $\mathscr{X}$ and $\varepsilon$ a Rademacher variable independent of $X$. Denote by $\left(X_{i}\right)$ (resp., $\left.\left(\varepsilon_{i}\right)\right)$ independent copies of $X($ resp., $\varepsilon)$. These two sequences are understood to be independent and constructed on different probability spaces. The following are equivalent:

(a) $\mathbf{E}\|X\|^{2}<\infty$ and $X \in \operatorname{CLT}(\mathscr{X})$;

(b) for almost every $\omega$ of the probability space supporting $X_{i}$ 's, the sequence $\left(n^{-1 / 2} \sum_{i \leqslant n} \varepsilon_{i} X_{i}(\omega)\right)$ converges in distribution in $\mathscr{X}$.

In either case, the limit of $\left(n^{-1 / 2} \sum_{i \leqslant n} \varepsilon_{i} X_{i}(\omega)\right)$ is distributed as $G(X)$, the Gaussian distribution with the same covariance structure as $X$.

Theorem 5. Assume that $B$ is of type 2 and the random element $\xi \in H_{\rho}^{\circ}(B)$ satisfies the following conditions:

(i) for every $t \in T, \mathbf{E} \xi(t)=0$ and $\mathbf{E}\|\xi\|_{B}^{2}<\infty$;

(ii) there exist a positive random variable $M$ and a function $\sigma$ : $[0,1] \rightarrow \mathbf{R}, \sigma(0)=0$, such that $\mathbf{E} M^{2}<\infty$ and

$$
\left\|\Delta_{h}^{2} \xi(t)\right\|_{B} \leqslant M \sigma(|h|), \quad \text { for all } t \in T, h \in C_{t}
$$

and

$$
\lim _{j \rightarrow \infty} \frac{\rho\left(2^{-j}\right)}{j^{1 / 2} \sigma\left(2^{-j}\right)}=\infty
$$

Then $\xi$ satisfies $\operatorname{CLT}\left(H_{\rho}^{o}(B)\right)$. 
P r o o f. We use the part (b) $\Rightarrow\left(\right.$ a) of Theorem 4 with $\mathscr{X}=H_{\rho}^{o}(B)$ and $X=\xi$. So we consider the randomized field

$$
\tilde{\zeta}_{n}^{\omega}(t):=n^{-1 / 2} \sum_{i=1}^{n} \varepsilon_{i} \xi_{i}(\omega, t), \quad t \in T,
$$

defined on the probability space $\Omega_{\varepsilon}$ supporting the sequence $\left(\varepsilon_{i}\right)$.

Since $B$ is of type 2 , it is clear from (i) that $\xi(t) \in \operatorname{CLT}(B)$ for every $t \in T$. So by the part (a) $\Rightarrow$ (b) of Theorem 4 with $\mathscr{X}=B$ and $X=\xi(t)$, we see that for almost every $\omega$ of the probability space $\Omega_{\xi}$ supporting the $\xi$ 's, $\varepsilon \xi(\omega, t) \in \operatorname{CLT}(B)$. It follows then from Lemma 2 that for almost every $\omega$, all the finite dimensional distributions of $\widetilde{\zeta}_{n}^{\omega}$ indexed by dyadic points of $T$ converge weakly.

The proof of the tightness relies on the sub-Gaussian behavior of Rademacher sums. More precisely, if $X$ is a $B$-valued Rademacher sum, then for every $s>0$,

$$
\mathbf{P}\left\{\|X\|_{B}>s\right\} \leqslant 2 \exp \left\{-\frac{s^{2}}{32 \mathbf{E}\|X\|_{B}^{2}}\right\} .
$$

To exploit (3.9), we need some uniform control (in $t, h$ and $n$ ) on the strong variance $\mathbf{E}_{\varepsilon}\left\|\Delta_{h}^{2} \widetilde{\zeta}_{n}^{\omega}(t)\right\|_{B}^{2}$. Denoting by $b$ the type 2 constant of the space $B$, we get from (3.7)

$$
\mathbf{E}\left\|\Delta_{h}^{2} \widetilde{\zeta}_{n}^{\omega}(t)\right\|_{B}^{2} \leqslant \frac{b^{2}}{n} \sum_{i=1}^{n} M_{i}^{2}(\omega) \sigma^{2}(|h|)
$$

where $M_{1}, \ldots, M_{n}$ are independent copies of the random variable $M$. By the strong law of large numbers, for almost every $\omega \in \Omega_{\xi}, c(\omega):=$ $\sup _{n \geqslant 1} b^{2} n^{-1} \sum_{i=1}^{n} M_{i}^{2}(\omega)$ is finite. Finally for almost every $\omega \in \Omega_{\xi}$,

$$
\mathbf{P}_{\varepsilon}\left\{\left\|\Delta_{h}^{2} \widetilde{\zeta}_{n}^{\omega}(t)\right\|_{B}>s\right\} \leqslant 2 \exp \left\{\frac{-s^{2}}{32 c(\omega) \sigma^{2}(|h|)}\right\}
$$

so the series condition of Corollary 1 with $\Psi(r)=2 \exp \left\{-r^{2} /(32 c(\omega))\right\}$ is satisfied under (3.8). Condition (i) of Theorem 2 following of the convergence of finite dimensional distributions, the almost sure tightness of $\widetilde{\zeta}_{n}^{\omega}$ is established and the proof is complete.

We next investigate the CLT in the space $H_{\rho}^{o}(T ; C(S))$, where $T=[0,1]$, $(S, \mathrm{~d})$ is a compact metric space and $C(S)$ denotes the Banach space of continuous real-valued functions on $S$. If $\delta$ is a pseudometric on $S$, then $N(S, \delta ; r)$ is the smallest number of open balls of radius $r>0$ in the pseudometric $\delta$ which form a covering of $T$. Denote further by $D(S ; \delta)$ the diameter of $(S, \delta)$ :

$$
D(S, \delta):=\sup \left\{\delta\left(s, s^{\prime}\right): s, s^{\prime} \in S\right\} .
$$

Theorem 6. Assume that the random element $\xi$ in $H_{\rho}^{o}(T ; C(S))$ is such that 
(i) for each $t \in T,(\xi(t, s), s \in S)$ satisfies the CLT in $C(S)$;

(ii) for some $s_{0} \in S,\left(\xi\left(t, s_{0}\right), t \in T\right)$ satisfies the $C L T$ in $H_{\rho}^{o}(T ; \mathbf{R})$;

(iii) there is a positive random variable $M, \mathbf{E} M^{2}<\infty$, and for each $h \in T$ there is a continuous pseudometric $\delta_{h}$ on $S$ such that for all $t \in T$, $s, s^{\prime} \in S$,

$$
\left|\Delta_{h}^{2}\left(\xi(t, s)-\xi\left(t, s^{\prime}\right)\right)\right| \leqslant M \delta_{h}\left(s, s^{\prime}\right) \quad \text { almost surely }
$$

and

$$
\sum_{j=1}^{\infty} \frac{1}{\rho\left(2^{-j}\right)} \int_{0}^{D_{j}} \ln ^{1 / 2}\left(2^{j} N_{j}(r)\right) d r<\infty
$$

where $D_{j}=D\left(S, \delta_{2^{-j}}\right)$ and $N_{j}(r)=N\left(S, \delta_{2^{-j}} ; r\right)$.

Then $\xi$ satisfies the CLT in $H_{\rho}^{o}(T ; C(S))$.

To establish Theorem 6 , we shall use the following lemma whose proof goes along the lines of [12, Theorem 11.2] and shall be omitted.

Lemma 3. Let $\phi$ be a Young function and let for each $l=1, \ldots, L$, $\left(X_{l}(t), t \in S\right)$ be a random process in $L_{1}(\Omega, \mathscr{F}, \mathbf{P})$ such that for all measurable sets $A$ and all $s, s^{\prime} \in S$ and $l=1, \ldots, L$

$$
\int_{A}\left|X_{l}(s)-X_{l}\left(s^{\prime}\right)\right| d \mathbf{P} \leqslant \mathbf{d}\left(s, s^{\prime}\right) \mathbf{P}(A) \phi^{-1}\left(\frac{1}{\mathbf{P}(A)}\right) .
$$

Then

$$
\mathbf{E} \max _{1 \leqslant l \leqslant L} \sup _{s, s^{\prime} \in S}\left|X_{l}(s)-X_{l}\left(s^{\prime}\right)\right| \leqslant 8 \int_{0}^{D(S, \mathbf{d})} \phi^{-1}(L N(S, \mathbf{d} ; \varepsilon)) d \varepsilon .
$$

Particularly, condition (3.12) is satisfied provided

$$
\left\|X_{l}(s)-X_{l}\left(s^{\prime}\right)\right\|_{\phi} \leqslant \mathbf{d}\left(s, s^{\prime}\right) .
$$

Proof. We use the part (b) $\Rightarrow$ (a) of Theorem 4 with $\mathscr{X}=$ $H_{\rho}^{o}(T ; C(S))$ and $X=\xi$. So we consider the randomized field

$$
\widetilde{\zeta}_{n}^{\omega}(t, s):=n^{-1 / 2} \sum_{i=1}^{n} \varepsilon_{i} \xi_{i}(\omega, t, s), \quad t \in T, \quad s \in S,
$$

defined on the probability space $\Omega_{\varepsilon}$ supporting the Rademacher sequence $\left(\varepsilon_{i}\right)$.

Part (a) $\Rightarrow$ (b) of Theorem 4 with $\mathscr{X}=C(S)$ and Lemma 2 give for almost every $\omega$, the weak convergence of all the finite dimensional distributions of $\widetilde{\zeta}_{n}^{\omega}$ indexed by dyadic points of $T$. Moreover condition (i) of Theorem 2 is fullfilled for almost every $\omega$. So it only remains to check condition (ii) in Theorem 2, that is prove that for almost every $\omega$, for every $\epsilon>0$,

$$
\lim _{J \rightarrow \infty} \sup _{n \geqslant 1} \mathbf{P}_{\varepsilon}\left\{\left\|\tilde{\zeta}_{n}^{\omega}-E_{J} \widetilde{\zeta}_{n}^{\omega}\right\|_{\rho}^{\text {seq }}>\epsilon\right\}=0,
$$


where the sequential norm is relative to the space $H(T, C(S), \rho(h))$, i.e.,

$$
\left\|\widetilde{\zeta}_{n}^{\omega}-E_{J} \widetilde{\zeta}_{n}^{\omega}\right\|_{\rho}^{\text {seq }}=\sup _{j>J} \frac{1}{\rho\left(2^{-j}\right)} \max _{v \in V_{j}} \sup _{s \in S}\left|\Delta_{2^{-j}}^{2} \widetilde{\zeta}_{n}^{\omega}(v, s)\right| .
$$

Now $\left\|\lambda_{j, v}\left(\widetilde{\zeta}_{n}^{\omega}\right)\right\|_{C(S)} \leqslant\left|\Delta_{2^{-j}}^{2} \widetilde{\zeta}_{n}^{\omega}\left(v, s_{0}\right)\right|+\sup _{s, s^{\prime} \in S}\left|\Delta_{2^{-j}}^{2} \widetilde{\zeta}_{n}^{\omega}(v, s)-\Delta_{2^{-j}}^{2} \widetilde{\zeta}_{n}^{\omega}\left(v, s^{\prime}\right)\right|$. Since $\left(\xi\left(t, s_{0}\right), t \in T\right)$ satisfies the CLT in $H_{\rho}^{o}(T ; \mathbf{R})$, Theorem 4 gives the tightness in this space of the sequence $\left(\tilde{\zeta}_{n}^{\omega}\left(\cdot, s_{0}\right)\right)$ for almost every $\omega$. Then the necessity of condition (ii) in Theorem 2 gives for almost every $\omega$

$$
\lim _{J \rightarrow \infty} \sup _{n \geqslant 1} \mathbf{P}_{\varepsilon}\left\{\sup _{j>J} \frac{1}{\rho\left(2^{-j}\right)} \max _{v \in V_{j}}\left|\widetilde{\zeta}_{n}^{\omega}\left(v, s_{0}\right)-E_{J} \widetilde{\zeta}_{n}^{\omega}\left(v, s_{0}\right)\right|>\epsilon\right\}=0 .
$$

Therefore (3.14) is reduced to

$$
\lim _{J \rightarrow \infty} \sup _{n \geqslant 1} T_{J, n}=0
$$

where

$$
T_{J, n}:=\sum_{j=J+1}^{\infty} \mathbf{P}_{\varepsilon}\left\{\max _{v \in V_{j}} \sup _{s, s^{\prime} \in S}\left|\Delta_{2-j}^{2} \widetilde{\zeta}_{n}^{\omega}(v, s)-\Delta_{2^{-j}}^{2} \widetilde{\zeta}_{n}^{\omega}\left(v, s^{\prime}\right)\right|>\epsilon \rho\left(2^{-j}\right)\right\} .
$$

Put

$$
I_{j, n}:=\mathbf{E}_{\varepsilon} \max _{v \in V_{j}} \sup _{s, s^{\prime} \in S}\left|\Delta_{2^{-j}}^{2} \widetilde{\zeta}_{n}^{\omega}(v, s)-\Delta_{2^{-j}}^{2} \widetilde{\zeta}_{n}^{\omega}\left(v, s^{\prime}\right)\right| .
$$

From the well-known fact that real Rademacher averages span a subspace isomorphic to $\ell^{2}$ in $L_{\phi_{2}}\left(\Omega_{\varepsilon}, \mathbf{P}_{\varepsilon}\right)$ (see, e.g., [12, p. 93]), we have a constant $C$ such that for each $j$, each $v \in V_{j}$ and almost every $\omega$

$$
\begin{aligned}
\left\|\Delta_{2^{-j}}^{2} \widetilde{\zeta}_{n}^{\omega}(v, s)-\Delta_{2^{-j}}^{2} \widetilde{\zeta}_{n}^{\omega}\left(v, s^{\prime}\right)\right\|_{\phi_{2}}^{2} & \leqslant \frac{C}{n} \sum_{i=1}^{n}\left|\Delta_{2^{-j}}^{2} \xi_{i}(\omega, v, s)-\Delta_{2^{-j}}^{2} \xi_{i}\left(\omega, v, s^{\prime}\right)\right|^{2} \\
& \leqslant \frac{C}{n} \sum_{i=1}^{n} M_{i}^{2}(\omega) \delta_{2^{-j}}^{2}\left(s, s^{\prime}\right)
\end{aligned}
$$

using (iii) and denoting by $M_{i}$ independent copies of $M$. By the strong law of large numbers in $\left(\Omega_{\xi}, \mathbf{P}_{\xi}\right)$, there is a positive constant $C(\omega)$ almost surely finite such that

$$
\left\|\Delta_{2^{-j}}^{2} \widetilde{\zeta}_{n}^{\omega}(v, s)-\Delta_{2^{-j}}^{2} \tilde{\zeta}_{n}^{\omega}\left(v, s^{\prime}\right)\right\|_{\phi_{2}} \leqslant C(\omega) \delta_{2^{-j}}\left(s, s^{\prime}\right) .
$$

This last estimate enables us to apply Lemma 3 with $X_{v}(s)=\Delta_{2^{-j}}^{2} \widetilde{\zeta}_{n}^{\omega}(v, s)$ and $L=\operatorname{card}\left(V_{j}\right)=2^{j}$ to obtain

$$
I_{j, n} \leqslant 8 \int_{0}^{D\left(S, C(\omega) \delta_{2-j}\right)} \ln ^{1 / 2}\left(2^{j} N\left(S, C(\omega) \delta_{2^{-j}}, \tau\right)\right) d \tau .
$$

Finally we have after the change of variable $\tau=r C(\omega)$,

$$
T_{J, n} \leqslant \sum_{j=J+1}^{\infty} \frac{8 C(\omega)}{\epsilon \rho\left(2^{-j}\right)} \int_{0}^{D_{j}} \ln ^{1 / 2}\left(2^{j} N_{j}(r)\right) d r
$$

whence (3.16) is satisfied for almost every $\omega$ due to assumption (3.11), so the proof is complete. 


\section{СПИСОК ЛИТЕРАТУРЫ}

1. Bonic R., Frampton J., Tromba A. $\Lambda$-manifolds. - J. Funct. Anal., 1969, v. 3, p. 310320 .

2. Ciesielski $Z$. On the isomorphisms of the spaces $H_{\alpha}$ and m. - Bull. Acad. Polon. Sci. Sér. Sci. Math. Astronom. Phys., 1960, v. 8, p. 217-222.

3. Ciesielski $Z$. Hölder conditions for realizations of Gaussian processes. - Trans. Amer. Math. Soc., 1961, v. 99, p. 403-413.

4. Enflo P. A counterexample to the approximation problem in Banach spaces. - Acta Math., 1973, v. 130, p. 309-317.

5. Delporte $J$. Fonctions aléatoires presque sûrement continues sur un intervalle fermé. Ann. Inst. H. Poincaré, Sect. B, 1964, v. 1, p. 111-215.

6. Erickson $R$. $V$. Lipschitz smoothness and convergence with applications to the central limit theorem for summation processes. - Ann. Probab., 1981, v. 9, № 5, p. 831-851.

7. Hamadouche D. Weak convergence of smoothed empirical process in Hölder spaces. Statist. Probab. Lett., 1998, v. 36, № 4, p. 393-400.

8. Hamadouche D. Invariance principles in Hölder spaces. - Portugal. Math., 2000, v. 57, № 2, p. 127-151.

9. Kerkyacharian $G$., Roynette $B$. Une démonstration simple des théorèmes de Kolmogorov, Donsker et Ito-Nisio. - C. R. Acad. Sci. Paris Sér. I Math., 1991, v. 312, № 11, p. $877-882$.

10. Lamperti J. On convergence of stochastic processes. - Trans. Amer. Math. Soc., 1962 , v. 104 , p. $430-435$.

11. Ledoux $M$. Sur une inégalité de H.P. Rosenthal et le théorème limite central dans les espaces de Banach. - Israel J. of Math., 1985, v. 50, № 4, p. 290-318.

12. Ledoux M., Talagrand M. Probability in Banach Spaces. Berlin: Springer-Verlag, 1991, $480 \mathrm{p}$.

13. Račkauskas A., Suquet Ch. Central limit theorem in Hölder spaces. - Probab. Math. Statist., 1999, v. 19, № 1, p. 133-152.

14. Račkauskas A., Suquet Ch. Random fields and central limit theorem in some generalized Hölder spaces. - Probability Theory and Mathematical Statistics: Proceedings of the Seventh Vilnius Conference (1998). Ed. by B. Grigelionis et al. Vilnius/Utrecht: TEV/VSP, 1999, p. 599-616.

15. Račkauskas A., Suquet $C h$. On the Hölderian functional central limit theorem for i.i.d. random elements in Banach space. - Limit Theorems of Probability and Statistics: Proceedings of the Fourth Hungarian Colloquium (Balatonlelle, 1999). Budapest: János Bolyai Math. Soc., 2002, p. 485-498.

16. Račkauskas A., Suquet Ch. Existence of versions and central limit theorems in Hölder topologies for Banach space valued random fields. - Publ. IRMA Lille, 2000, v. 52, article II.

17. Račkauskas A., Suquet Ch. Hölder versions of Banach space valued random fields. Georgian Math. J., 2001, v. 8, № 2, p. 347-362.

18. Semadeni $Z$. Schauder bases in Banach spaces of continuous functions. - Lecture Notes in Math., 1982, v. 918, p. 1-136.

19. Singer I. Bases in Banach Spaces. II. Berlin-New York: Springer-Verlag, 1981, 880 p.

20. Suquet Ch. Tightness in Schauder decomposable Banach spaces. - Amer. Math. Soc. Transl. Ser. 2, 1999, v. 193, p. 201-224. 